\title{
Matrix Quasinorms Induced by Maximal and Minimal Vector Norms
}

\section{Jong-Do Park}

Department of Mathematics and Research Institute for Basic Sciences, Kyung Hee University, Seoul 130-701, Republic of Korea

Correspondence should be addressed to Jong-Do Park; mathjdpark@khu.ac.kr

Received 6 July 2016; Accepted 28 September 2016

Academic Editor: Hans G. Feichtinger

Copyright (C) 2016 Jong-Do Park. This is an open access article distributed under the Creative Commons Attribution License, which permits unrestricted use, distribution, and reproduction in any medium, provided the original work is properly cited.

In the set of all vector norms in $\mathbb{C}^{n}$, there exist maximal and minimal complex norms which coincide with the real Euclidean norm in $\mathbb{R}^{n}$. The purpose of this paper is to introduce new quasinorms defined on complex matrices. These two matrix quasinorms are induced by maximal and minimal complex vector norms. We also prove the dual relation between these two quasinorms.

\section{Introduction}

The standard Euclidean norm in $\mathbb{R}^{n}\left(\right.$ or $\left.\mathbb{C}^{n}\right)$ is

$$
\|x\|:=\sqrt{\sum_{j=1}^{n}\left|x_{j}\right|^{2}},
$$

where $x=\left(x_{1}, \ldots, x_{n}\right) \in \mathbb{R}^{n}$ (or $\mathbb{C}^{n}$ ). We could easily extend this vector norm to matrices, just by taking a matrix $Z=$ $\left(z_{i j}\right)_{1 \leq i \leq m, 1 \leq j \leq n}$ as a vector in $\mathbb{R}^{m n}$ (or $\mathbb{C}^{m n}$ ). This natural extension is called the Frobenius norm $\|Z\|_{F}$ (also called Hilbert-Schmidt norm or Schur norm) defined by

$$
\|Z\|_{F}:=\sqrt{\sum_{j=1}^{m} \sum_{k=1}^{n}\left|z_{j k}\right|^{2}}=\sqrt{\operatorname{tr}\left(Z \bar{Z}^{T}\right)} .
$$

In $\mathbb{C}^{n}$, there are another two well-known norms $L(\cdot)$ and $N^{*}(\cdot)$,called the maximal norm and the minimal norm introduced by Siciak [1] and Hahn-Pflug [2], respectively. For $z \in \mathbb{C}^{n}$, the explicit forms of $L(z)$ and $N^{*}(z)$ are given by

$$
L(z)=\sqrt{\|z\|^{2}+\sqrt{\|z\|^{4}-|z \cdot z|^{2}}},
$$

$$
N^{*}(z)=\sqrt{\frac{1}{2}\left(\|z\|^{2}+|z \cdot z|\right)},
$$

where $z \cdot w:=\sum_{j=1}^{n} z_{j} w_{j}$ for $z, w \in \mathbb{C}^{n}$.

It is known that $L(z)$ and $N^{*}(z)$ have the following properties [1-3]:

(i) $L(\cdot)$ and $N^{*}(\cdot)$ are norms.

(ii) $N^{*}(z)=L(z)=\|z\|$ for all $z \in \mathbb{R}^{n}$.

(iii) $N^{*}(z) \leq\|z\| \leq L(z)$ for all $z \in \mathbb{C}^{n}$.

(iv) They are dual in the sense that $|z \bullet w| \leq L(z) N^{*}(w)$ for all $z, w \in \mathbb{C}^{n}$.

In fact, $L(z)$ and $N^{*}(z)$ are maximal and minimal norms satisfying (ii) and (iii). The Bergman kernel for the ball $\mathbb{B}_{*}:=$ $\left\{z \in \mathbb{C}^{n}: N_{*}(z)<1\right\}$ was computed explicitly in [4]. Moreover, recently many papers deal with function theoretic problems on $\mathbb{B}_{*}$ in [5-9].

In this paper, similarly to the Frobenius norm, we extend $L(\cdot)$ and $N^{*}(\cdot)$ to complex matrices satisfying (ii), (iii), and (iv). At first, we show that these two extensions are quasinorms (see Theorem 4 ). The second result is the duality of $L(\cdot)$ and $N^{*}(\cdot)$ like Hölder inequality (see Theorem 5 ). Also we construct $N_{p}(Z)$ for $p \geq 1$. If $p=1$, then $N_{1}(Z)=$ $N^{*}(Z)$ and $\lim _{p \rightarrow \infty} N_{p}(Z)=L(Z)$. Finally, we proved the dual relation between $N_{p}$ and $N_{q}$ when $1 / p+1 / q=1$ (see Corollary 6 and Theorem 7). 


\section{Statements of Main Results}

In 1981, Siciak [1] found the existence of complex maximal extension of $\|\cdot\|$ called the Lie norm $L(z)$ for $z=\left(z_{1}, \ldots, z_{n}\right) \in$ $\mathbb{C}^{n}$. The maximal norm $L(z)$ satisfies

(i) $L(x)=\|x\|$ for $x \in \mathbb{R}^{n}$,

(ii) $N(z) \leq L(z)$ for any complex norm $N(z)$ with $N(x)=$ $\|x\|$ for $x \in \mathbb{R}^{n}$.

In addition, the explicit form of $L(z)$ is known as in (3). In fact, the ball $\left\{z \in \mathbb{C}^{n}: L(z)<1\right\}$ is called the Lie ball which is a classical bounded symmetric domain of type IV.

For the minimal complex extension, it is known that such a norm does not exist, since there is a sequence of complex extensions of $\|\cdot\|$ which converges to 0 at certain points. One can see a counterexample in [2]. In 1988, Hahn and Pflug [2] constructed the complex minimal extension of $\|\cdot\|$ called the minimal norm $N^{*}(z)$ in a slightly different sense as follows:

(i) $N^{*}(x)=\|x\|$ for $x \in \mathbb{R}^{n}$.

(ii) $N^{*}(z) \leq N(z)$ for any complex norm $N(z)$ with $N(x)=\|x\|$ for $x \in \mathbb{R}^{n}$ and $N(z) \leq\|z\|$ for $z \in \mathbb{C}^{n}$.

Moreover, the explicit form of $N^{*}(z)$ is known as in (4). The Bergman kernel for the ball $\left\{z \in \mathbb{C}^{n}: N^{*}(z)<1\right\}$ was computed explicitly in [4].

In [3], Morimoto and Fujita proved the following relation between $L(z)$ and $N^{*}(z)$ when $z \in \mathbb{C}^{n}$.

Proposition 1 (see [3]). Let $L(z)$ and $N^{*}(z)$ be norms defined as in (3) and (4) for $z \in \mathbb{C}^{n}$. Then, for $z, w \in \mathbb{C}^{n}$,

(i) $|z \cdot w| \leq L(z) N^{*}(w)$,

(ii) $L(z)=\sup \left\{|z \cdot w|: N^{*}(w)=1\right\}$.

Now we deal with the generalization of $N^{*}(\cdot)$ and $L(\cdot)$ when $z$ is a complex matrix. In 2002, Youssfi [9] introduced the natural generalization of $N^{*}(\cdot)$ from complex vectors to complex matrices. Let $\mathscr{M}$ be the set of all $m \times n$ complex matrices. For $Z=\left(z_{j k}\right)_{1 \leq j \leq m, 1 \leq k \leq n} \in \mathscr{M}$, we define the row vectors $Z^{(j)}$ by

$$
Z^{(j)}=\left(z_{j 1}, z_{j 2}, \ldots, z_{j n}\right),
$$

where $j=1,2, \ldots, m$. The Bergman kernel and the Szegö kernel for the ball $\left\{Z \in \mathscr{M}: N^{*}(Z)<1\right\}$ have been computed by using proper holomorphic liftings (see [9]).

Definition 2. For $Z=\left(z_{j k}\right)_{1 \leq j \leq m, 1 \leq k \leq n} \in \mathscr{M}$, we define $N^{*}(Z)$ by

$$
\begin{aligned}
N^{*}(Z) & :=\sqrt{\frac{1}{2} \sum_{j=1}^{m}\left(\left\|Z^{(j)}\right\|^{2}+\left|Z^{(j)} \cdot Z^{(j)}\right|\right)} \\
& =\sqrt{\frac{1}{2}\left(\sum_{j=1}^{m} \sum_{k=1}^{n}\left|z_{j k}\right|^{2}+\sum_{j=1}^{m}\left|\sum_{k=1}^{n} z_{j k}^{2}\right|\right)} .
\end{aligned}
$$

Similarly, we define $L(Z)$ and $M(Z)$ for $Z \in \mathscr{M}$ as follows.
Definition 3. For $Z=\left(z_{j k}\right)_{1 \leq j \leq m, 1 \leq k \leq n} \in \mathscr{M}$, we define $L(Z)$ and $M(Z)$ by

$L(Z)$

$$
\begin{aligned}
& :=\sqrt{\sum_{j=1}^{m}\left\|Z^{(j)}\right\|^{2}+\sqrt{\left(\sum_{j=1}^{m}\left\|Z^{(j)}\right\|^{2}\right)^{2}-\left(\sum_{j=1}^{m}\left|Z^{(j)} \cdot Z^{(j)}\right|\right)^{2}}} \\
& =\sqrt{\sum_{j=1}^{m} \sum_{k=1}^{n}\left|z_{j k}\right|^{2}+\sqrt{\left(\sum_{j=1}^{m} \sum_{k=1}^{n}\left|z_{j k}\right|^{2}\right)^{2}-\left(\sum_{j=1}^{m}\left|\sum_{k=1}^{n} z_{j k}^{2}\right|\right)^{2}}}
\end{aligned}
$$

$M(Z)$

$$
\begin{aligned}
& :=\sqrt{\sum_{j=1}^{m}\left\|Z^{(j)}\right\|^{2}-\sqrt{\left(\sum_{j=1}^{m}\left\|Z^{(j)}\right\|^{2}\right)^{2}-\left(\sum_{j=1}^{m}\left|Z^{(j)} \cdot Z^{(j)}\right|\right)^{2}}} \\
& =\sqrt{\sum_{j=1}^{m} \sum_{k=1}^{n}\left|z_{j k}\right|^{2}-\sqrt{\left(\sum_{j=1}^{m} \sum_{k=1}^{n}\left|z_{j k}\right|^{2}\right)^{2}-\left(\sum_{j=1}^{m}\left|\sum_{k=1}^{n} z_{j k}^{2}\right|\right)^{2}}} .
\end{aligned}
$$

If $m=1$, then $L(Z)$ and $N^{*}(Z)$ are the Lie norm and the minimal norm in $\mathbb{C}^{n}$, respectively, and if $n=1$, then $L(Z)=N^{*}(Z)$ is the Euclidean norm in $\mathbb{C}^{m}$. Moreover, it is easily proved that the inequality

$$
\frac{1}{\sqrt{2}}\|Z\|_{F} \leq N^{*}(Z) \leq\|Z\|_{F} \leq L(Z) \leq \sqrt{2}\|Z\|_{F}
$$

holds, where the Frobenius norm $\|Z\|_{F}$ is defined as in (2).

At first we prove that extensions $L(Z)$ and $N^{*}(Z)$ of these norms to complex matrices are quasinorms of $\mathscr{M}$.

Theorem 4. For $Z \in \mathscr{M}, L(Z)$ and $N^{*}(Z)$ are quasinorms. Precisely, for $Z, W \in M$ we have

(i) $L(Z+W) \leq \sqrt{m}\{L(Z)+L(W)\}$,

(ii) $N^{*}(Z+W) \leq \sqrt{m}\left\{N^{*}(Z)+N^{*}(W)\right\}$. follows.

The generalization of Proposition 1(i) will be proved as

Theorem 5. For $Z, W \in \mathscr{M}$, we have

$$
\sum_{j=1}^{m}\left|\sum_{k=1}^{n} z_{j k} w_{j k}\right| \leq L(Z) N^{*}(W) .
$$

We also generalize Proposition 1(ii) for complex matrices as follows. 
Corollary 6. For $Z \in \mathscr{M}$, we have

$$
\begin{aligned}
& \text { (i) } \min _{1 \leq j \leq m} L\left(Z^{(j)}\right) \\
& \leq \sup \left\{\sum_{j=1}^{m}\left|Z^{(j)} \bullet W^{(j)}\right|: 1 \leq \sum_{j=1}^{m} N^{*}\left(W^{(j)}\right) \leq \sqrt{m}\right\} \\
& \leq \sqrt{m} L(Z) \\
& \text { (ii) } \min _{1 \leq j \leq m} L\left(Z^{(j)}\right) \\
& \leq \sup \left\{\sum_{j=1}^{m}\left|Z^{(j)} \bullet W^{(j)}\right|: \sum_{j=1}^{m} N^{*}\left(W^{(j)}\right)=1\right\} \\
& \leq L(Z) .
\end{aligned}
$$

For $p \geq 1$ and $Z \in \mathscr{M}$, we define

$$
N_{p}(Z):=\left\{\frac{1}{2}\left(\sum_{j=1}^{m} L\left(Z^{(j)}\right)^{p}+M\left(Z^{(j)}\right)^{p}\right)\right\}^{1 / p},
$$

where

$$
\begin{aligned}
& M\left(Z^{(j)}\right) \\
& \quad:=\sqrt{\left\|Z^{(j)}\right\|^{2}-\sqrt{\left(\left\|Z^{(j)}\right\|^{2}\right)^{2}-\left(\left|Z^{(j)} \cdot Z^{(j)}\right|\right)^{2}}} .
\end{aligned}
$$

One can easily see that $N_{1}(Z)=N^{*}(Z)$ and $\lim _{p \rightarrow \infty} N_{p}(Z)=$ $L(Z)$. Then, we finally proved the following.

Theorem 7. For $Z \in \mathscr{M}$, we have

$$
\begin{aligned}
& \text { (i) } \min _{1 \leq j \leq m} N_{p}\left(Z^{(j)}\right) \\
& \leq \sup \left\{\sum_{j=1}^{m}\left|Z^{(j)} \bullet W^{(j)}\right|: 1 \leq \sum_{j=1}^{m} N_{q}\left(W^{(j)}\right) \leq m^{1 / p}\right\} \\
& \leq m^{1 / p} N_{p}(Z) \\
& \text { (ii) } \min _{1 \leq j \leq m} N_{p}\left(Z^{(j)}\right) \\
& \leq \sup \left\{\sum_{j=1}^{m}\left|Z^{(j)} \cdot W^{(j)}\right|: \sum_{j=1}^{m} N_{q}\left(W^{(j)}\right)=1\right\} \\
& \leq N_{p}(Z)
\end{aligned}
$$

where $1 / p+1 / q=1$

Remark 8. If $m=1$, then Corollary 6 and Theorem 7 are identical to previous results proved by Morimoto and Fujita [3].

\section{Proofs}

Throughout this section, it is convenient to define

$$
\begin{aligned}
& a_{j}:=\sum_{k=1}^{n}\left|z_{j k}\right|^{2}, \\
& b_{j}:=\left|\sum_{k=1}^{n} z_{j k}^{2}\right|, \\
& c_{j}:=\sum_{k=1}^{n}\left|w_{j k}\right|^{2}, \\
& d_{j}:=\left|\sum_{k=1}^{n} w_{j k}^{2}\right|, \\
& e_{j}:=\sum_{k=1}^{n}\left|z_{j k}+w_{j k}\right|^{2}, \\
& f_{j}:=\left|\sum_{k=1}^{n}\left(z_{j k}+w_{j k}\right)^{2}\right| .
\end{aligned}
$$

Note that $a_{j} \geq b_{j} \geq 0, c_{j} \geq d_{j} \geq 0$, and $e_{j} \geq f_{j} \geq 0$.

Lemma 9. Assume that $x_{1}, \ldots, x_{m}, y_{1}, \ldots, y_{m}$ satisfy $x_{j} \geq$ $y_{j} \geq 0$ for all $j=1, \ldots, m$. Then, we have

(i) $\sum_{j=1}^{m} \sqrt{x_{j}^{2}-y_{j}^{2}} \leq \sqrt{\left(\sum_{j=1}^{m} x_{j}\right)^{2}-\left(\sum_{j=1}^{m} y_{j}\right)^{2}}$,

$$
\text { (ii) } \begin{aligned}
& \sqrt{\sum_{j=1}^{m} x_{j}+\sqrt{\left(\sum_{j=1}^{m} x_{j}\right)^{2}-\left(\sum_{j=1}^{m} y_{j}\right)^{2}}} \\
& \leq \sum_{j=1}^{m} \sqrt{x_{j}+\sqrt{x_{j}^{2}-y_{j}^{2}}}
\end{aligned}
$$

Proof. (i) If we apply Cauchy-Schwarz inequality, then we have

$$
\begin{aligned}
\sum_{j=1}^{m} \sqrt{x_{j}^{2}-y_{j}^{2}} & =\sum_{j=1}^{m} \sqrt{x_{j}+y_{j}} \sqrt{x_{j}-y_{j}} \\
& \leq \sqrt{\sum_{j=1}^{m}\left(x_{j}+y_{j}\right) \sum_{j=1}^{m}\left(x_{j}-y_{j}\right)} \\
& =\sqrt{\left(\sum_{j=1}^{m} x_{j}\right)^{2}-\left(\sum_{j=1}^{m} y_{j}\right)^{2}} .
\end{aligned}
$$


(ii) Note that

$$
\begin{gathered}
\left(\sum_{j=1}^{m} \sqrt{\left.x_{j}+\sqrt{x_{j}^{2}-y_{j}^{2}}\right)^{2}}\right. \\
\quad-\left\{\sum_{j=1}^{m} x_{j}+\sqrt{\left(\sum_{j=1}^{m} x_{j}\right)^{2}-\left(\sum_{j=1}^{m} y_{j}\right)^{2}}\right\} \\
=2 \sum_{j \neq l} \sqrt{x_{j}+\sqrt{x_{j}^{2}-y_{j}^{2}}} \sqrt{x_{l}+\sqrt{x_{l}^{2}-y_{l}^{2}}} \\
+\sum_{j=1}^{m} \sqrt{x_{j}^{2}-y_{j}^{2}}-\sqrt{\left(\sum_{j=1}^{m} x_{j}\right)^{2}-\left(\sum_{j=1}^{m} y_{j}\right)^{2}} .
\end{gathered}
$$

The last term is estimated as follows:

$$
\begin{aligned}
& \sqrt{\left(\sum_{j=1}^{m} x_{j}\right)^{2}-\left(\sum_{j=1}^{m} y_{j}\right)^{2}} \\
& =\sqrt{\sum_{j=1}^{m}\left(x_{j}^{2}-y_{j}^{2}\right)+2 \sum_{j \neq l}\left(x_{j} x_{l}-y_{j} y_{l}\right)} \\
& \leq \sum_{j=1}^{m} \sqrt{x_{j}^{2}-y_{j}^{2}}+\sqrt{2 \sum_{j \neq l}\left(x_{j} x_{l}-y_{j} y_{l}\right)} .
\end{aligned}
$$

It follows that

$$
\begin{aligned}
& \left(\sum_{j=1}^{m} \sqrt{x_{j}+\sqrt{x_{j}^{2}-y_{j}^{2}}}\right)^{2} \\
& -\left\{\sum_{j=1}^{m} x_{j}+\sqrt{\left(\sum_{j=1}^{m} x_{j}\right)^{2}-\left(\sum_{j=1}^{m} y_{j}\right)^{2}}\right\} \\
& \geq 2 \sum_{j \neq l} \sqrt{x_{j}+\sqrt{x_{j}^{2}-y_{j}^{2}}} \sqrt{x_{l}+\sqrt{x_{l}^{2}-y_{l}^{2}}} \\
& -\sqrt{2 \sum_{j \neq l}\left(x_{j} x_{l}-y_{j} y_{l}\right)} \geq 2 \sum_{j \neq l} \sqrt{x_{j} x_{l}}-\sqrt{2 \sum_{j \neq l} x_{j} x_{l}} \\
& >(2-\sqrt{2}) \sqrt{\sum_{j \neq l} x_{j} x_{l}}>0,
\end{aligned}
$$

since $y_{j} y_{l} \geq 0$ for all $j, l$. The proof of (ii) is finished.

3.1. Proof of Theorem 4(i). For $j=1,2, \ldots, m$, we define the row vectors $Z^{(j)}$ and $W^{(j)}$ by

$$
\begin{aligned}
Z^{(j)} & =\left(z_{j 1}, z_{j 2}, \ldots, z_{j n}\right), \\
W^{(j)} & =\left(w_{j 1}, w_{j 2}, \ldots, w_{j n}\right) .
\end{aligned}
$$

Since $L(z)$ is a norm with $z \in \mathbb{C}^{n}$, for each $j=1,2, \ldots, m$, we have

$$
L\left(Z^{(j)}+W^{(j)}\right) \leq L\left(Z^{(j)}\right)+L\left(W^{(j)}\right)
$$

so that

$$
\sum_{j=1}^{m} L\left((Z+W)^{(j)}\right) \leq \sum_{j=1}^{m} L\left(Z^{(j)}\right)+\sum_{j=1}^{m} L\left(W^{(j)}\right) .
$$

Now we will obtain the upper bound of

$$
\sum_{j=1}^{m} L\left(Z^{(j)}\right)+\sum_{j=1}^{m} L\left(W^{(j)}\right)
$$

By Cauchy-Schwarz inequality and Lemma 9(i), we have

$$
\begin{aligned}
& \sum_{j=1}^{m} L\left(Z^{(j)}\right) \\
& \quad=\sum_{j=1}^{m} \sqrt{\sum_{k=1}^{n}\left|z_{j k}\right|^{2}+\sqrt{\left(\sum_{k=1}^{n}\left|z_{j k}\right|^{2}\right)^{2}-\left|\sum_{k=1}^{n} z_{j k}^{2}\right|^{2}}} \\
& \quad=\sum_{j=1}^{m} \sqrt{a_{j}+\sqrt{a_{j}^{2}-b_{j}^{2}} \leq \sqrt{m} \sqrt{\sum_{j=1}^{m} a_{j}+\sum_{j=1}^{m} \sqrt{a_{j}^{2}-b_{j}^{2}}}} \\
& \quad \leq \sqrt{m} \sqrt{\sum_{j=1}^{m} a_{j}+\sqrt{\left(\sum_{j=1}^{m} a_{j}\right)^{2}-\left(\sum_{j=1}^{m} b_{j}\right)^{2}}} \\
& \quad=\sqrt{m} L(Z) .
\end{aligned}
$$

Similarly, we have

$$
\sum_{j=1}^{m} L\left(W^{(j)}\right) \leq \sqrt{m} L(W) .
$$

Thus, we see that

$$
\sum_{j=1}^{m} L\left(Z^{(j)}\right)+\sum_{j=1}^{m} L\left(W^{(j)}\right) \leq \sqrt{m}(L(Z)+L(W)) .
$$

By Lemma 9(ii), we have

$$
\begin{aligned}
L(Z+W) & =\sqrt{\sum_{j=1}^{m} e_{j}+\sqrt{\left(\sum_{j=1}^{m} e_{j}\right)^{2}-\left(\sum_{j=1}^{m} f_{j}\right)^{2}}} \\
& \leq \sum_{j=1}^{m} \sqrt{e_{j}+\sqrt{e_{j}^{2}-f_{j}^{2}}} \\
& =\sum_{j=1}^{m} L\left((Z+W)^{(j)}\right) .
\end{aligned}
$$

Combining (24), (28), and (29), we obtain that $L(\cdot)$ is a quasinorm. 
3.2. Proof of Theorem 4(ii). Now we show that $N^{*}(\cdot)$ is a quasinorm. Note that

$$
\begin{aligned}
N^{*}(Z+W) & =\sqrt{\frac{1}{2} \sum_{j=1}^{m}\left(e_{j}+f_{j}\right)}<\sum_{j=1}^{m} \sqrt{\frac{1}{2}\left(e_{j}+f_{j}\right)} \\
& =\sum_{j=1}^{m} N^{*}\left((Z+W)^{(j)}\right) .
\end{aligned}
$$

Since $N^{*}(Z)$ is a norm when $m=1$ in [3], we have

$$
\sum_{j=1}^{m} N^{*}\left((Z+W)^{(j)}\right) \leq \sum_{j=1}^{m} N^{*}\left(Z^{(j)}\right)+\sum_{j=1}^{m} N^{*}\left(W^{(j)}\right) .
$$

By Cauchy-Schwarz inequality,

$$
\begin{aligned}
\sum_{j=1}^{m} N^{*}\left(Z^{(j)}\right) & =\sum_{j=1}^{m} \sqrt{\frac{1}{2}\left(a_{j}+b_{j}\right)} \\
& \leq \sqrt{m} \sqrt{\frac{1}{2} \sum_{j=1}^{m}\left(a_{j}+b_{j}\right)}=\sqrt{m} N^{*}(Z)
\end{aligned}
$$

and similarly

$$
\sum_{j=1}^{m} N^{*}\left(W^{(j)}\right) \leq \sqrt{m} N^{*}(W)
$$

Combining the above inequalities, we obtain that $N^{*}(\cdot)$ is also a quasinorm.

3.3. Proof of Theorem 5. We will prove that Proposition 1(i) holds also for the matrices. By Proposition 1(i), we have

$$
\begin{aligned}
\left|\sum_{k=1}^{n} z_{j k} w_{j k}\right| & \leq L\left(Z^{(j)}\right) N^{*}\left(Z^{(j)}\right) \\
& =\sqrt{a_{j}+\sqrt{a_{j}^{2}-b_{j}^{2}}} \times \sqrt{\frac{1}{2}\left(c_{j}+d_{j}\right)}
\end{aligned}
$$

for each $j=1, \ldots, m$. By Cauchy-Schwarz inequality and Lemma 9(i), we have

$$
\begin{gathered}
\sum_{j=1}^{m}\left|\sum_{k=1}^{n} z_{j k} w_{j k}\right| \leq \sum_{j=1}^{m} \sqrt{a_{j}+\sqrt{a_{j}^{2}-b_{j}^{2}}} \times \sqrt{\frac{1}{2}\left(c_{j}+d_{j}\right)} \\
\leq \sqrt{\sum_{j=1}^{m}\left(a_{j}+\sqrt{a_{j}^{2}-b_{j}^{2}}\right) \sqrt{\sum_{j=1}^{m} \frac{1}{2}\left(c_{j}+d_{j}\right)}} \\
\leq \sqrt{\sum_{j=1}^{m} a_{j}+\sqrt{\left(\sum_{j=1}^{m} a_{j}\right)^{2}-\left(\sum_{j=1}^{m} b_{j}\right)^{2}}} \\
\times \sqrt{\frac{1}{2}\left(\sum_{j=1}^{m} c_{j}+\sum_{j=1}^{m} d_{j}\right)}=L(Z) N^{*}(W) .
\end{gathered}
$$

3.4. Proof of Corollary 6. Note that

$$
N^{*}(W) \leq \sum_{j=1}^{m} N^{*}\left(W^{(j)}\right) \leq \sqrt{m} N^{*}(W) .
$$

If $1 \leq \sum_{j=1}^{m} N^{*}\left(W^{(j)}\right) \leq \sqrt{m}$, then $N^{*}(W) \leq \sqrt{m}$ by (36). So, by Theorem 5 ,

$$
\sum_{j=1}^{m}\left|Z^{(j)} \cdot W^{(j)}\right| \leq \sqrt{m} L(Z)
$$

so that

$$
\begin{aligned}
& \sup \left\{\sum_{j=1}^{m}\left|Z^{(j)} \cdot W^{(j)}\right|: 1 \leq \sum_{j=1}^{m} N^{*}\left(W^{(j)}\right) \leq \sqrt{m}\right\} \\
& \quad \leq \sqrt{m} L(Z) .
\end{aligned}
$$

On the other hand, we have

$$
\begin{aligned}
& \sup \left\{\sum_{j=1}^{m}\left|Z^{(j)} \bullet W^{(j)}\right|: 1 \leq \sum_{j=1}^{m} N^{*}\left(W^{(j)}\right) \leq \sqrt{m}\right\} \\
& =\sup \left\{\sum_{j=1}^{m} k_{j}\left|Z^{(j)} \bullet\left(\frac{W}{k_{j}}\right)^{(j)}\right|: 1 \leq \sum_{j=1}^{m} k_{j}\right. \\
& \left.\leq \sqrt{m}, N^{*}\left(\left(\frac{W}{k_{j}}\right)^{(j)}\right)=1\right\},
\end{aligned}
$$

where $k_{j}:=N^{*}\left(W^{(j)}\right)$ for $1 \leq j \leq m$. By Proposition 1(ii), the last term is greater than or equal to

$$
\begin{aligned}
& \inf \left\{\sum_{j=1}^{m} k_{j} L\left(Z^{(j)}\right): 1 \leq \sum_{j=1}^{m} k_{j} \leq \sqrt{m}\right\} \\
& \geq \min _{1 \leq j \leq m} L\left(Z^{(j)}\right) \sum_{j=1}^{m} k_{j} \geq \min _{1 \leq j \leq m} L\left(Z^{(j)}\right) .
\end{aligned}
$$

It follows that

$$
\begin{aligned}
& \min _{1 \leq j \leq m} L\left(Z^{(j)}\right) \leq \sup \left\{\sum_{j=1}^{m}\left|Z^{(j)} \cdot W^{(j)}\right|: 1\right. \\
& \left.\leq \sum_{j=1}^{m} N^{*}\left(W^{(j)}\right) \leq \sqrt{m}\right\} .
\end{aligned}
$$

The proof of (i) of Corollary 6 is finished. The proof of (ii) is similar to that of (i).

3.5. Proof of Theorem 7. We use the following inequalities for vectors. 
Proposition 10 (see [3]). For $z, w \in \mathbb{C}^{n}$, we have

(i) $2|z \cdot w| \leq L(z) L(w)+M(z) M(w)$

(ii) $N_{p}(z)=\sup \left\{|z \bullet w|: N_{q}(w)=1\right\}$, where $1 / p+1 / q=1$.

By Proposition 10(i), we have

$$
\begin{gathered}
2 \sum_{j=1}^{m}\left|Z^{(j)} \cdot W^{(j)}\right| \leq \sum_{j=1}^{m} L\left(Z^{(j)}\right) L\left(W^{(j)}\right)+M\left(Z^{(j)}\right) \\
\cdot M\left(W^{(j)}\right) \leq\left(\sum_{j=1}^{m} L\left(Z^{(j)}\right)^{p}+M\left(Z^{(j)}\right)^{p}\right)^{p} \\
\cdot\left(\sum_{j=1}^{m} L\left(W^{(j)}\right)^{q}+M\left(W^{(j)}\right)^{q}\right)^{q},
\end{gathered}
$$

where $1 / p+1 / q=1$. From $2=2^{1 / p+1 / q}$, we obtain

$$
\sum_{j=1}^{m}\left|Z^{(j)} \cdot W^{(j)}\right| \leq N_{p}(Z) N_{q}(W) .
$$

The Hölder inequality tells us that if $x_{j} \geq 0$ for all $1 \leq j \leq m$, then

$$
\left(\sum_{j=1}^{m} x_{j}\right)^{1 / q} \leq \sum_{j=1}^{m} x_{j}^{1 / q} \leq m^{1 / p}\left(\sum_{j=1}^{m} x_{j}\right)^{1 / q} .
$$

If we substitute $x_{j}=(1 / 2)\left(L\left(W^{(j)}\right)^{p}+M\left(W^{(j)}\right)\right)$ in (44), then we obtain

$$
N_{q}(W) \leq \sum_{j=1}^{m} N_{q}\left(W^{(j)}\right) \leq m^{1 / p} N_{q}(W) .
$$

Now we can prove Theorem 7 similarly to the proof of Corollary 6. From (43) and (45), if $1 \leq \sum_{j=1}^{m} N_{q}\left(W^{(j)}\right) \leq m^{1 / p}$, then

$$
\sum_{j=1}^{m}\left|Z^{(j)} \cdot W^{(j)}\right| \leq m^{1 / p} N_{p}(Z) .
$$

Similarly to the proof of Corollary 6 , we write $k_{j}:=$ $N_{q}\left(W^{(j)}\right)$ for $1 \leq j \leq m$. Then, using (45) and Proposition 10(ii), we have

$$
\begin{aligned}
& \sup \left\{\sum_{j=1}^{m}\left|Z^{(j)} \bullet W^{(j)}\right|: 1 \leq \sum_{j=1}^{m} N_{q}\left(W^{(j)}\right) \leq m^{1 / p}\right\} \\
& =\sup \left\{\sum_{j=1}^{m} k_{j}\left|Z^{(j)} \cdot\left(\frac{W}{k_{j}}\right)^{(j)}\right|: 1 \leq \sum_{j=1}^{m} k_{j}\right.
\end{aligned}
$$

$$
\begin{aligned}
& \left.\leq m^{1 / p}, N_{q}\left(\left(\frac{W}{k_{j}}\right)^{(j)}\right)=1\right\} \\
& \geq \inf \left\{\sum_{j=1}^{m} k_{j} N_{p}\left(Z^{(j)}\right): 1 \leq \sum_{j=1}^{m} k_{j} \leq \sqrt{m}\right\} \\
& \geq \min _{1 \leq j \leq m} N_{p}\left(Z^{(j)}\right) \sum_{j=1}^{m} k_{j} \geq \min _{1 \leq j \leq m} N_{p}\left(Z^{(j)}\right) .
\end{aligned}
$$

It follows that

$$
\begin{aligned}
& \min _{1 \leq j \leq m} N_{p}\left(Z^{(j)}\right) \leq \sup \left\{\sum_{j=1}^{m}\left|Z^{(j)} \bullet W^{(j)}\right|: 1\right. \\
& \left.\leq \sum_{j=1}^{m} N_{q}\left(W^{(j)}\right) \leq m^{1 / p}\right\} .
\end{aligned}
$$

From (46) and (48), we complete the proof of (i) of Theorem 7. The proof of (ii) is similar to that of (i).

\section{Competing Interests}

The author declares that there is no conflict of interests regarding the publication of this paper.

\section{Acknowledgments}

This work was supported by the National Research Foundation of Korea (NRF-2015R1D1A1A01060295).

\section{References}

[1] J. Siciak, "Extremal plurisubharmonic functions in $\mathbb{C}^{n}$," Annales Polonici Mathematici, vol. 39, pp. 175-211, 1981.

[2] K. T. Hahn and P. Pflug, "On a minimal complex norm that extends the real Euclidean norm," Monatshefte für Mathematik, vol. 105, no. 2, pp. 107-112, 1988.

[3] M. Morimoto and K. Fujita, "Between Lie norm and dual Lie norm," Tokyo Journal of Mathematics, vol. 24, no. 2, pp. 499-507, 2001.

[4] K. Oeljeklaus, P. Pflug, and E. H. Youssfi, “The Bergman kernel of the minimal ball and applications," Annales de l'Institut Fourier, vol. 47, no. 3, pp. 915-928, 1997.

[5] J. Gonessa, "Duality of Fock spaces with respect to the minimal norm," Archiv der Mathematik, vol. 100, no. 5, pp. 439-447, 2013.

[6] J. Gonessa and K. Zhu, "A sharp norm estimate for weighted Bergman projections on the minimal ball," New York Journal of Mathematics, vol. 17A, pp. 101-112, 2011.

[7] N. Viêt Anh and E. H. Youssfi, "Lipschitz estimates for the $\bar{\partial}$ equation on the minimal ball," Michigan Mathematical Journal, vol. 49, no. 2, pp. 299-323, 2001.

[8] J.-D. Park, "Uniform extendibility of the Bergman kernel for generalized minimal balls," Journal of Function Spaces, vol. 2015, Article ID 919465, 8 pages, 2015. 
[9] E. H. Youssfi, "Proper holomorphic liftings and new formulas for the Bergman and Szegö kernels," Studia Mathematica, vol. 152, no. 2, pp. 161-186, 2002. 


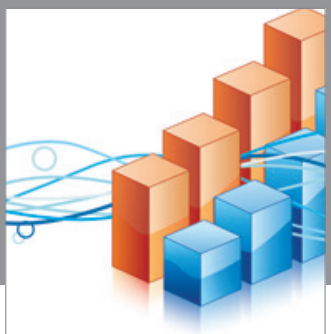

Advances in

Operations Research

vatem alat4

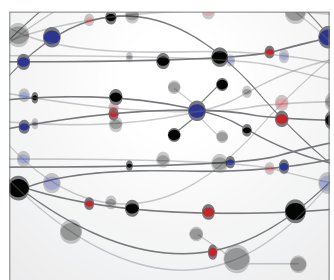

\section{The Scientific} World Journal
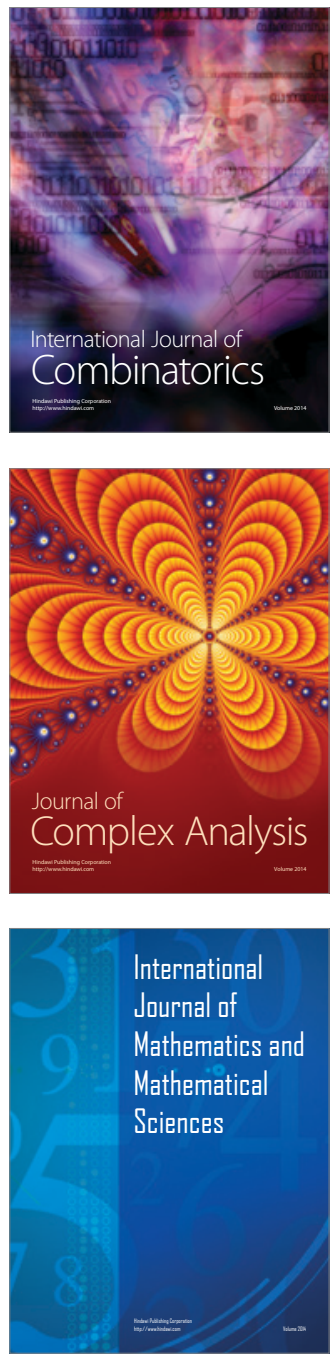
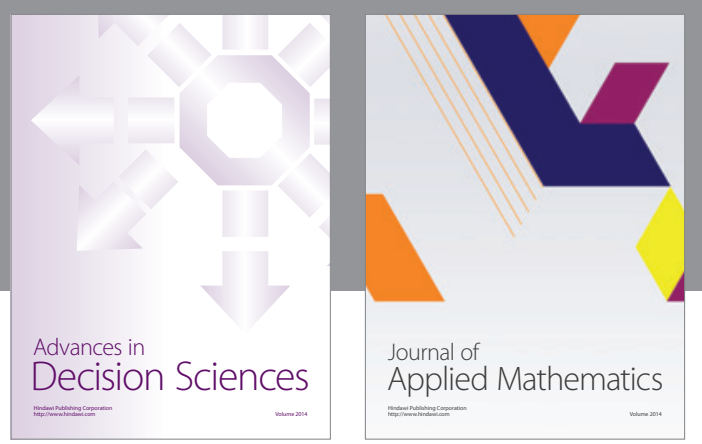

Algebra

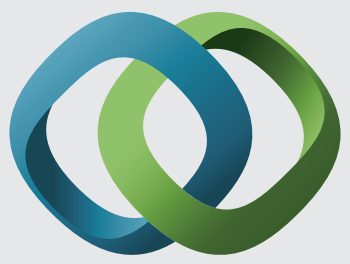

\section{Hindawi}

Submit your manuscripts at

http://www.hindawi.com
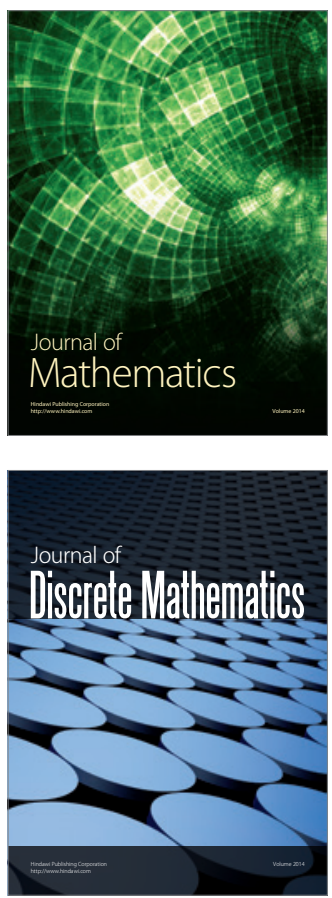

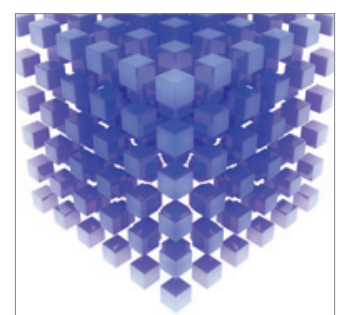

Mathematical Problems in Engineering
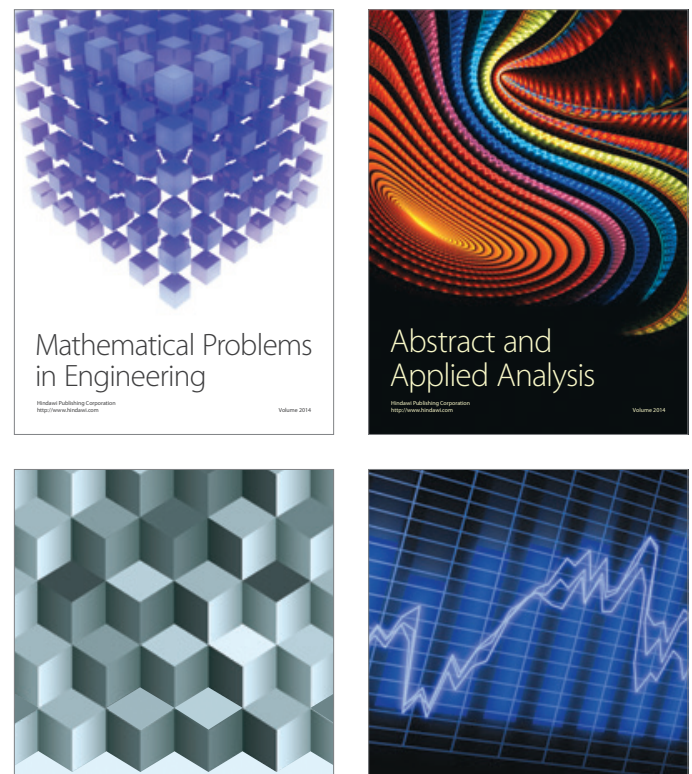

Journal of

Function Spaces

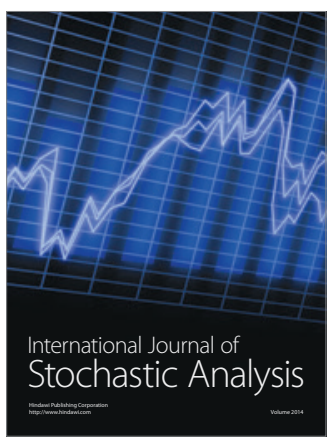

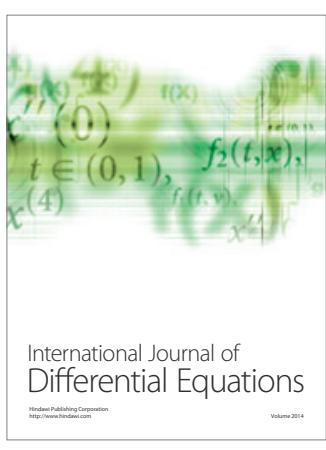
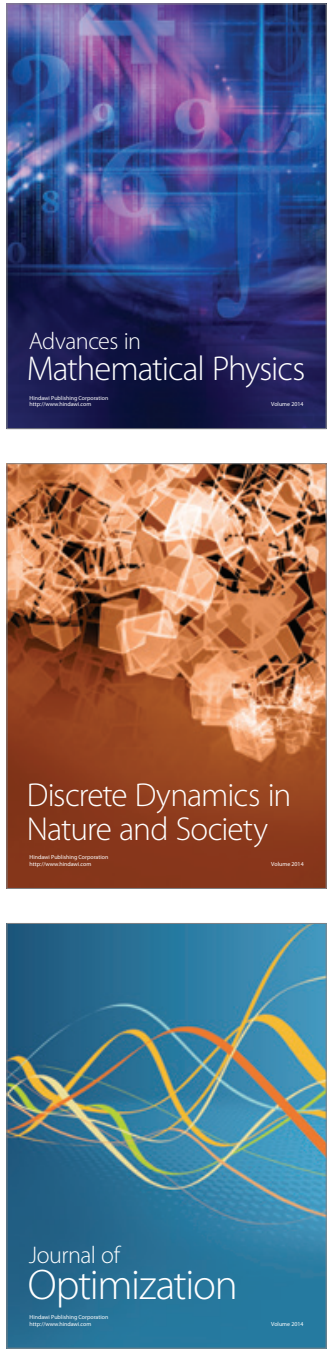